\title{
Open charm in nuclear matter at finite temperature
}

\author{
L. Tolós, ${ }^{1}$ A. Ramos, ${ }^{2}$ and T. Mizutani ${ }^{3}$ \\ ${ }^{1}$ Frankfurt Institute for Advanced Studies, J. W. Goethe-Universität, Ruth-Moufang-Str. 1, D-60438 Frankfurt am Main, Germany \\ ${ }^{2}$ Departament d'Estructura i Constituents de la Matèria, Universitat de Barcelona, Diagonal 647, E-08028 Barcelona, Spain \\ ${ }^{3}$ Department of Physics, Virginia Polytechnic Institute and State University, Blacksburg, Virginia 24061, USA
}

(Received 14 October 2007; published 22 January 2008)

\begin{abstract}
We study the properties of open-charm mesons $(D$ and $\bar{D})$ in nuclear matter at finite temperature within a self-consistent coupled-channel approach. The meson-baryon interactions are adopted from a type of broken SU(4) $s$-wave Tomozawa-Weinberg term supplemented by an attractive scalar-isoscalar interaction. The inmedium solution at finite temperature incorporates Pauli blocking effects, mean-field binding on all the baryons involved, and $\pi$ and open-charm meson self-energies in a self-consistent manner. In the $D N$ sector, the $\Lambda_{c}$ and $\Sigma_{c}$ resonances, generated dynamically at 2593 and $2770 \mathrm{MeV}$ in free space, remain close to their free-space position while acquiring a remarkable width due to the thermal smearing of Pauli blocking as well as from the nuclear matter density effects. As a result, the $D$ meson spectral density shows a single pronounced peak for energies close to the $D$ meson free-space mass that broadens with increasing matter density with an extended tail particularly toward lower energies. The $\bar{D}$ potential shows a moderate repulsive behavior coming from the dominant $I=1$ contribution of the $\bar{D} N$ interaction. The low-density theorem is, however, not a good approximation for the $\bar{D}$ self-energy in spite of the absence of resonance-hole contributions close to threshold in this case. We speculate the possibility of $D$-mesic nuclei as well as discuss some consequences for the $J / \Psi$ suppression in heavy-ion collisions, in particular for the future CBM experiment at FAIR.
\end{abstract}

DOI: 10.1103/PhysRevC.77.015207

\section{INTRODUCTION}

The interest in the open and hidden charmed mesons within the context of relativistic nucleus-nucleus collisions was triggered about 20 years ago. More specifically, the suppression of the $J / \Psi$ production was predicted as a rather clear signature of the formation of quark-gluon plasma (QGP) in ultrarelativistic central nucleus-nucleus collisions in Ref. [1]. According to its authors, a Debye-type color screening in the gluon exchanges blocks the formation of charmonium $(c \bar{c})$ bound states. Then, starting about 10 years later, the NA50 and NA60 Collaborations [see, for example, Refs. [2-5], in the CERN Super Proton Synchrotron (SPS) fixed target experiments] actually claimed to have observed such a suppression in $\mathrm{Pb}+\mathrm{Pb}$ collisions at $\approx 160 \mathrm{~A} \mathrm{GeV}$. On the start of the Brookhaven Relativistic Heavy Ion Collider (RHIC) heavy nucleus collider a little more than 6 years ago with, say, the central collision of $\mathrm{Au}+\mathrm{Au}$ at $\sqrt{s_{N N}}=200 \mathrm{GeV}$, a new set of exciting results has gradually come out, such as an apparent energy independence of the $J / \Psi$ suppression [6] as compared with the SPS result with $\sqrt{s_{N N}}=17 \mathrm{GeV}$. Firmly establishing the origin of this charmonium suppression as due to the formation of the QGP appears to need more careful analyses of the data. However, if formed in such ultrarelativistic collisions, the QGP would correspond to the one with a rather high temperature: $T>T_{c}$, where the critical temperature extracted from recent quantum chromodynamics (QCD) lattice simulation [7] is $T_{c} \approx 175 \mathrm{MeV}$ with a low-baryon number density $\rho_{B}$ (or chemical potential $\mu_{B}$ ), supposedly similar to the situation during the initial Big Bang period. This part of the $T-\mu_{B}$ phase diagram of the hadronic/partonic matter will continue to be the major subject of further intense activities at RHIC as well as at the CERN LHC facility (expected at $\sqrt{s_{N N}}>$ $5 \mathrm{TeV}$ for $\mathrm{Pb}+\mathrm{Pb}$ ).
PACS number(s): 12.38.Lg, 14.20.Lq, 14.20.Jn, 21.65.-f

Equally interesting as well as important is the somewhat complementary region of the phase diagram that is characterized by a moderate temperature but with large $\rho_{B}$. According to recent lattice simulations (see, for example, Ref. [8]), here a highly compressed hadronic matter gets transformed into a dense partonic matter (or strongly interacting QGP) where the boundary of the two phases is characterized by a first-order phase transition, as opposed to the above-mentioned RHIC-type hadron $\leftrightarrow$ QGP transition which the calculation has found as a smooth crossover (see also a so-called Polyakov loop extended NJL (PNJL) approach to the subject [9]). The CBM (compressed baryon matter) experiment of the FAIR project at GSI aims at investigating an important portion of this moderate $T$ and large $\mu_{B}$ part of the phase diagram by a high-intensity beam of, for example, uranium nuclei of up to $35 \mathrm{~A} \mathrm{GeV}$ that overlaps with the SPS energy. In this way one may expect to study possible modifications of the properties of various mesons in dense baryonic matter. In particular, because the charmed mesons produced at FAIR will not be at high energies but could be close to threshold, their medium modification may be significative. This should apply equally to the production of open-charm mesons such as $D$ and $\bar{D}$ as well as to hidden charmed mesons: charmonia. For the latter, one will be able to study the possible suppression and regeneration of the $J / \Psi$ at moderate energies by mechanisms that may be of conventional hadronic origin or due to deconfinement but different from the high $T$ (QGP) color screening scheme proposed in Ref. [1] and being sought by the far higher energy RHIC accelerator, as stated earlier.

Our present interest is in relativistic heavy-ion collisions that fit into some part of the domain of the phase diagram covered by the FAIR project. In particular, we focus on hadronic approaches to the in-medium modification of the 
$D(\bar{D})$ mesons that may (i) enter the explanation of the possible $J / \Psi$ suppression in relativistic nucleus-nucleus collisions, see, for example, Ref. [10], with special interest in the FAIR energies; (ii) provide a theoretical support for an anticipated open-charm enhancement again within the FAIR energies [11], an issue that was triggered by the NA50 Collaboration but was not recognized by the NA60 result, see Ref. [12]; and (iii) infer possible $D^{0}, D^{-}, \bar{D}^{0}$ bound states in heavy nuclei such as $\mathrm{Pb}$ [13].

Here we should stress that all these interpretations/ predictions are based on the possible attraction felt by the $D(\bar{D})$ mesons which could lead to their mass reduction in the nuclear medium [13-16]. For example, within these mostly hadronic pictures, the $J / \Psi$ absorption by collision with nucleons and mesons was suggested to take place at little or no extra cost of energy due to the lowering of the threshold for $D \bar{D}$ pairs, facilitating reactions of the $J / \Psi$ with comoving mesons, such as $J / \Psi \pi \rightarrow D \bar{D}$. In a similar manner, processes such as $J / \Psi N \rightarrow \bar{D} Y_{c}$, where $Y_{c}$ is one of the charmed baryons, may proceed more easily. A critical and detailed review of these mean-field approaches was made in Ref. [17], which eventually points to the necessity of performing a coupled-channel meson-baryon scattering in nuclear medium due to strong coupling among the $D N$ and other meson-baryon channels with same quantum numbers. Hence, in the present article, we pursue a coupled-channel study on the spectral properties of the open-charm $D$ and $\bar{D}$ mesons in nuclear matter at finite temperatures. In this regard, we want to remark that kinetic equilibrium assumed in the corresponding heavy-ion reactions to introduce a well-defined temperature may yet to be firmly established, although, as pointed out in Ref. [11], nonequilibrium transport equation methods appear to support the thermalization picture [18-20] at SPS energies. To set the basis, we should mention here earlier prototypes of the present study. First, a coupled-channel approach based on a set of separable meson-baryon interactions was adopted with an underlying $\mathrm{SU}(3)$ symmetry among the $u, d$, and $c$ quarks (thus excluding the strangeness related channels). After model parameters were fixed to reproduce the position and width of the $\Lambda_{c}(2593)$, it was applied to study the in-medium spectral function of the $D$ meson for a zero-temperature nuclear matter environment [21]. This was later extended to finite temperatures [22]. Next, based on a SU(4) scheme broken by the masses of the exchanged vector mesons (the charmed ones in particular), hence including also the channels with strangeness [23], an effective meson-baryon interaction, of lowest order in both chiral and heavy-quark symmetries, was introduced to study the $D$ mesons in nuclear matter at zero temperature $[17,24]$.

To continue and complete this sequence, in the present article we are extending the result of Ref. [17] to study the $D$ and $\bar{D}$ mesons in nuclear matter of up to twice the normal density and having a temperature from zero to $150 \mathrm{MeV}$. A couple of extra additions in the present work include (a) an explicit consideration of the nuclear mean-field binding effect on all the baryons involved in the coupled channel, inclusive of those that are strange and charmed, and (b) the study of the in-medium $\bar{D}$ (note that Ref. [24] also looked at this aspect). Equipped with those tools and ingredients, we go beyond the result of
Refs. [13-16] and try to determine the continuous in-medium spectral (or mass) distribution of $D$ and $\bar{D}$ and show that the former deviates significantly from the $\delta$ function-type spike at the free-space mass or at the value shifted to elsewhere. Also we obtain optical potentials for $D$ and $\bar{D}$. These are important ameliorations in view of the points (i)-(iii) stated above.

The organization of the present article goes as follows: in Sec. II we develop the formalism and ingredients on which the calculation in the present work is based. Section III is devoted to the presentation and discussion of the results. Finally, in Sec. IV we draw our conclusions and give final remarks pertaining to the present and future works.

\section{OPEN-CHARM MESONS IN NUCLEAR MATTER AT FINITE TEMPERATURE}

Our objective in this section is to obtain the $D$ and $\bar{D}$ in-medium self-energy by solving the corresponding multichannel $T$-matrix equation. Then, the obtained self-energy is used to find the $D(\bar{D})$ spectral function in an isosymmetric nuclear matter at finite temperature. This is done by extending the procedure found in Ref. [17] to a nonzero temperature environment by the procedure adopted in Ref. [22]. As mentioned in Sec. I, we shall also introduce the binding effect to all the baryons involved by the nuclear matter mean field.

\section{A. Coupled meson-baryon channels in free space}

The first step toward our goal is to obtain the free space $T$ matrices for the coupled meson-baryon system involving $D N(\bar{D} N)$. We shall briefly summarize it as discussed in Ref. [17]. These matrices follow the standard multichannel scattering (integral) equation,

$$
T=V+V G T,
$$

where $V$ is a symmetric matrix consisting of a set of mesonbaryon transition interactions (potentials). They are obtained from the tree-level $s$-wave contribution to the meson-baryon scattering and will be specified later. As shown in Ref. [25,26], the kernel of the equation for $s$-wave interaction can be factorized in the on-mass-shell ansatz, leaving the four-momentum integration only in the two-particle meson-baryon propagators. These quantities, often called loop functions, form a diagonal matrix $G$. They are divergent and thus need to be regularized. In the present work the cut-off method is adopted as it is more appropriate than dimensional methods when dealing with particles in a medium, as done in Refs. [27,28] where it is applied in the study of $\bar{K}$ in nuclear matter.

The consequence of the on-shell ansatz is a set of linear algebraic equations whose solution now reads,

$$
T=[1-V G]^{-1} V,
$$

which is practically equivalent to the so-called $N / D$ method [25].

The meson-baryon transition interaction $V$ is characterized here by the channel quantum numbers, charm $C$, strangeness $S$, and isospin $I$. We implicitly fix the first two quantum numbers and label $V$ explicitly by $I$. For each $(C, S)$ fixed, the coupled-channel elements of the symmetric matrix $V$ 
of a given isospin $I$ are specified as $V_{i j}^{I}$ for a transition $i \leftrightarrow j$. For $(C=1, S=0), i$ and $j$ run through $\pi \Sigma_{c}(2589)$, $D N(2810), \eta \Lambda_{c}$ (2835), $K \Xi_{c}(2960), K \Xi_{c}^{\prime}(3071), D_{s} \Lambda(3085)$, and $\eta^{\prime} \Lambda_{c}(3245)$ for the $I=0$ sector, and $\pi \Lambda_{c}(2425)$, $\pi \Sigma_{c}$ (2589), $D N(2810), K \Xi_{c}(2960), \eta \Sigma_{c}(3005), K \Xi_{c}^{\prime}(3071)$, $D_{s} \Sigma(3160)$, and $\eta^{\prime} \Sigma_{c}(3415)$ for the $I=1$ sector. In the case $(C=-1, S=0)$, there is only a single channel, $\bar{D} N(2810)$, for each isospin $I=0$ and $I=1$ value. We note that, in the above description, the value in the parentheses denotes the channel threshold in $\mathrm{MeV}$.

The concrete form for the matrix elements of $V$ comes from the $s$-wave Tomozawa-Weinberg (T-W) term as the zero-range limit of the lowest-order interaction of the SU(4) pseudoscalar meson and $1 / 2^{+}$ground-state baryon multiplets based on the universal vector-meson coupling hypothesis equipped with the extended KSFR condition (see Ref. [17] for details):

$$
\begin{aligned}
V_{i j}^{I}(\sqrt{s})= & -\frac{\kappa C_{i j}}{4 f^{2}}\left(2 \sqrt{s}-M_{i}-M_{j}\right) \\
& \times\left(\frac{M_{i}+E_{i}}{2 M_{i}}\right)^{1 / 2}\left(\frac{M_{j}+E_{j}}{2 M_{j}}\right)^{1 / 2} .
\end{aligned}
$$

The coupling strength $C_{i j}$ derives from the SU(4) symmetry for the $i \leftrightarrow j$ transition; $\sqrt{s}$ is the center-of-mass energy; $f=1.15 f_{\pi}$, where the value of $f$ has been adopted from Ref. [26], and $M_{i}$ and $M_{j}$ as well as $E_{i}$ and $E_{j}$ are the masses and energies of baryons in channels $i$ and $j$, respectively. The breaking of the SU(4) symmetry in the T-W interaction through the physical hadron masses is mostly eminent in the reduction factor $\kappa$, which is unity for transitions $i \leftrightarrow j$, driven by uncharmed vector-meson exchanges $\left(\rho, \omega, \phi, K^{*}\right)$ but is equal to $\kappa_{c}=\left(\bar{m}_{V} / \bar{m}_{V}^{c}\right)^{2} \approx 1 / 4$ for charmed vectormeson exchanges such as $D^{*}$ and $D_{s}^{*}$, where $\bar{m}_{V}\left(\bar{m}_{V}^{c}\right)$ is the mass of the typical uncharmed (charmed) exchanged vector meson. The transition coefficients $\tilde{C}_{i j} \equiv \kappa C_{i j}$, which are symmetric with respect to the indices, are listed in Tables I and II of Ref. [17] for the $D N$ sector. For the $\bar{D} N$ system, $\tilde{C}_{\bar{D} N \rightarrow \bar{D} N}^{I=0}=0$ and $\tilde{C}_{\bar{D} N \rightarrow \bar{D} N}^{I=1}=-2$. The reader is reminded of the same situation for the $K N$ coupling strengths due to $S U$ (3) symmetry. The T-W vector interaction is supplemented by a scalar-isoscalar attraction, which turns out to be important in kaon condensate studies (see Ref. [16,29]). The $s$-wave projection of this interaction is equal to

$$
V_{\Sigma}(\sqrt{s})=-\frac{\Sigma_{D N}}{f_{D}^{2}}\left(\frac{M_{N}+E}{2 M_{N}}\right),
$$

and it is independent of the $C$ and $I$ specification in the present context. Here, $f_{D}$ is the $D(\bar{D})$ meson weak decay constant and $\Sigma_{D N}$ is the strength of this interaction. Note that for simplicity we introduce this only in the diagonal $D(\bar{D}) N$ interaction. The most recent determination of $f_{D}=157 \mathrm{MeV}$ may be found in Ref. [30]. As for the value of $\Sigma_{D N}$, we simply follow what a QCD sum-rule [15] and a nuclear mean-field approach of Ref. [16] have suggested, and estimate it conservatively as $\Sigma_{D N} \approx 2000 \mathrm{MeV}$. Because we can aim only at qualitative estimates, we set $f_{D} \sim 200 \mathrm{MeV}$ and determine the strength of the scalar-isoscalar interaction to be $\Sigma=\Sigma_{D N} / f_{D}^{2}=$ $0.05 \mathrm{MeV}^{-1}$. We also accommodate the case where no such attraction is added, hence $\Sigma_{D N}=0$. Based on the above interactions, the multichannel transition $T$ matrices are solved such that the momentum cut-off $\Lambda$ is fixed to reproduce the position and the width of the $I=0 \quad \Lambda_{c}$ (2593) resonance. The parameters for the two cases adopted from Ref. [17] are used in the present work, model A: $f=1.15 f_{\pi}, \quad \Sigma=$ $0.05 \mathrm{MeV}^{-1}, \Lambda=727 \mathrm{MeV}$; and model B: $f=1.15 f_{\pi}, \Sigma=$ $0 \mathrm{MeV}^{-1}, \Lambda=787 \mathrm{MeV}$. These two model interactions produce a resonance in $I=1$ channel whose position and width are $2770 \mathrm{MeV}, 20 \mathrm{MeV}$ (model A), and $2795 \mathrm{MeV}, 20 \mathrm{MeV}$ (model B), respectively, close to the nominal $\Sigma_{c}(2800)$ [31].

\section{B. Coupled meson-baryon interaction in finite-temperature nuclear matter with mean-field binding}

The properties of the $D(\bar{D})$ mesons in nuclear matter at finite temperature and with mean-field binding are obtained by incorporating the corresponding medium modifications in the loop function matrix $G$ only. That is, we assume that the interaction matrix $V$ stays unchanged in medium. In Eq. (3), the two square-rooted factors come from the baryon spinor normalization, hence should stay more or less the same. The finite-temperature nuclear mean-field binding makes the in-medium baryon masses $M_{i}^{*}(T)$ to be shifted from their free space values $M_{i}$, so the second factor in the expression should be different in medium. We nevertheless retain the vacuum mass values in this interaction as we have estimated the effect to be at most a few percentages in magnitude. For the same reason the scalar-isoscalar interaction Eq. (4) remains unchanged. By implicitly understanding that $G$ and $T$ are now to be interpreted as medium modified, the same multichannel algebraic equation Eq. (2) is to be solved.

Let us now describe in detail the medium and temperature modifications. First, the baryons in the coupled channel, namely the nucleon, $\Lambda, \Sigma, \Lambda_{c}$, and $\Sigma_{c}$, change both their mass and energy-momentum relations due to the finite-temperature mean-field binding effect. We have adopted a temperaturedependent Walecka-type $\sigma-\omega$ model to account for this change; see, for example, Ref. [32]. Within this model, the nucleon energy spectrum in mean-field approximation is obtained from

$$
E_{N}(\vec{p}, T)=\sqrt{\vec{p}^{2}+M_{N}^{*}(T)^{2}}+\Sigma^{v}
$$

with the vector potential $\Sigma^{v}$ and the effective mass $M_{N}^{*}(T)$ given by

$$
\begin{aligned}
\Sigma^{v} & =\left(\frac{g_{v}}{m_{v}}\right)^{2} \rho \\
M_{N}^{*}(T) & =M_{N}-\Sigma^{s}, \quad \text { with } \Sigma^{s}=\left(\frac{g_{s}}{m_{s}}\right)^{2} \rho_{s},
\end{aligned}
$$

where $m_{s}$ and $m_{v}$ are the meson masses, whereas $g_{s}$ and $g_{v}$ are the density-dependent scalar and vector coupling constants, respectively. These constants are obtained by reproducing the energy per particle of symmetric nuclear matter at $T=0$ coming from a Dirac-Brueckner-Hartree-Fock calculation (see Table 10.9 of Ref. [33]). The ordinary nuclear matter (Lorentz) vector density $(\rho)$ and (Lorentz) scalar density $\left(\rho_{s}\right)$ are obtained from the corresponding vector $n(\vec{p}, T)$ and 
scalar $n_{s}(\vec{p}, T)$ density distributions, defined in terms of the Fermi-Dirac function as

$$
\begin{aligned}
n(\vec{p}, T) & =\frac{1}{1+\exp \left[\left(E_{N}(\vec{p}, T)-\mu\right) / T\right]}, \\
n_{s}(\vec{p}, T) & =\frac{M^{*}(T) n(\vec{p}, T)}{\sqrt{\vec{p}^{2}+M^{*}(T)^{2}}},
\end{aligned}
$$

by momentum integration, namely

$$
\rho=\frac{4}{(2 \pi)^{3}} \int d^{3} p n(\vec{p}, T),
$$

and similarly for $\rho_{s}$. As may be clear from the above development, $E_{N}(\vec{p}, T), M_{N}^{*}(T)$ and the chemical potential $\mu$ are obtained simultaneously and self-consistently for given $\rho$ and for the corresponding values of $g_{s}$ and $g_{v}$. The values of the nucleon scalar and vector potentials in nuclear matter at $\rho_{0}=0.16 \mathrm{fm}^{-3}$ and at $T=0$ are $\Sigma^{s}=356 \mathrm{MeV}$ and $\Sigma^{v}=278 \mathrm{MeV}$.

The hyperon $(Y)$ as well as the charmed baryon $\left(Y_{c}\right)$ masses and energy spectra can be easily inferred from those for the nucleon as

$$
E_{Y_{(c)}}(\vec{p}, T)=\sqrt{\vec{p}^{2}+M_{Y_{(c)}}^{*}(T)^{2}}+\Sigma_{Y_{(c)}}^{v},
$$

where

$$
\begin{aligned}
\Sigma_{Y_{(c)}}^{v} & =\frac{2}{3}\left(\frac{g_{v}}{m_{v}}\right)^{2} \rho=\frac{2}{3} \Sigma^{v} \\
M_{Y_{(c)}}^{*}(T) & =M_{Y_{(c)}}-\Sigma_{Y_{(c)}}^{s}=M_{Y_{(c)}}-\frac{2}{3}\left(\frac{g_{s}}{m_{s}}\right)^{2} \rho_{s} \\
& =M_{Y_{(c)}}-\frac{2}{3}\left[M_{N}-M_{N}^{*}(T)\right] .
\end{aligned}
$$

Here we have assumed that the $\sigma$ and $\omega$ fields couple only to the $u$ and $d$ quarks, as in Ref. [34], so the scalar and vector coupling constants for hyperons and charmed baryons are:

$$
g_{v}^{Y_{(c)}}=\frac{2}{3} g_{v}, \quad g_{s}^{Y_{(c)}}=\frac{2}{3} g_{s} .
$$

We note that the quark-meson coupling (QMC) calculations of Ref. [34], performed at $T=0$, obtained a somewhat smaller scalar potential (about half the present one) for the $\Lambda_{(c)}$ and $\Sigma_{(c)}$ baryons due to the inclusion of an effective coupling for each baryon species, $C_{j}(\tilde{\sigma})$, where " $j$ " is the label for baryons. This factor was introduced to mimic the baryon structure. To the best of our knowledge, no temperature effects have been studied within this framework.

The potential at zero momentum $V=E_{Y(c)}(\vec{p}=0)-$ $M_{Y(c)}$ for different baryon species obtained in the present work is shown in Fig. 1 as a function of the density for different temperatures. Obviously, the potential for hyperons and charmed baryons follows the simple light quark counting rule as compared with the nucleon potential: $V_{Y_{(c)}}=2 / 3 V_{N}$. The attraction at $\rho=\rho_{0}$ and $T=0 \mathrm{MeV}$ is about $-50 \mathrm{MeV}$, the size of which gets reduced as temperature increases, turning even into repulsion, especially at higher densities. This behavior results from the fact that the temperature-independent vector potential takes over the strongly temperature-dependent scalar potential, which decreases with temperature.

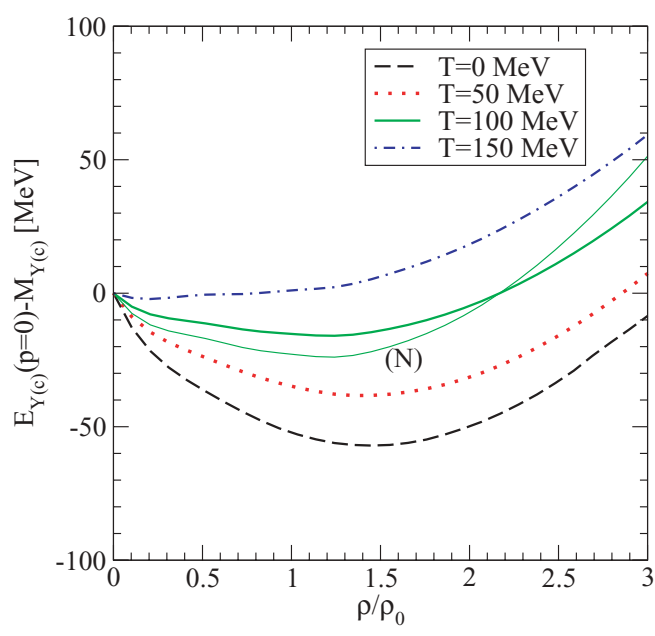

FIG. 1. (Color online) The potential at zero momentum for the hyperons $(Y=\Lambda, \Sigma)$ and charmed baryons $\left(Y_{c}=\Lambda_{c}, \Sigma_{c}\right)$ as a function of the density for different temperatures. The thin solid line displays the nucleon potential at $T=100 \mathrm{MeV}$.

The second medium effect is the Pauli exclusion principle acting on the nucleon in the intermediate $D(\bar{D}) N$ loop function. This is implemented by replacing the single freenucleon propagator in the loop function by the corresponding in-medium one:

$$
\begin{aligned}
G_{N}\left(p_{0}, \vec{p}, T\right)= & \frac{1-n(\vec{p}, T)}{p_{0}-E_{N}(\vec{p}, T)+\mathrm{i} \varepsilon} \\
& +\frac{n(\vec{p}, T)}{p_{0}-E_{N}(\vec{p}, T)-\mathrm{i} \varepsilon},
\end{aligned}
$$

where the effect of the temperature is contained in the nucleon Fermi-Dirac distributions and single-particle energies.

The third medium effect is the dressing of the mesons, due to their interactions with the surrounding nucleons in the course of propagating through nuclear matter. In particular, we will consider the dressing of pions and $D(\bar{D})$ mesons. The reason for not doing so for other mesons will be stated below.

The meson dressing is represented by the in-medium meson self-energy $\Pi_{i}\left(q_{0}, \vec{q}, T\right)$, where $i=D, \bar{D}, \pi$ in the present case. This quantity appears in the corresponding in-medium single meson propagator:

$$
D_{i}\left(q_{0}, \vec{q}, T\right)=\frac{1}{q_{0}^{2}-\vec{q}^{2}-m_{i}^{2}-\Pi_{i}\left(q_{0}, \vec{q}, T\right)} .
$$

In the Lehmann integral representation, the meson propagator may be expressed in terms of the spectral function $S_{i}\left(q_{0}, \vec{q}, T\right)$ as

$$
D_{i}\left(q_{0}, \vec{q}, T\right)=\int_{0}^{\infty} \frac{S_{i}(\omega, \vec{q}, T)}{q_{0}-\omega+\mathrm{i} \varepsilon} d \omega-\int_{0}^{\infty} \frac{S_{\bar{i}}(\omega, \vec{q}, T)}{q_{0}+\omega-\mathrm{i} \varepsilon} d \omega,
$$

where $\bar{i}$ is the antiparticle to meson $i$. Then we easily relate the self-energy and spectral function as

$$
\begin{aligned}
S_{i}\left(q_{0}, \vec{q}, T\right) & =-\frac{1}{\pi} \operatorname{Im} D_{i}\left(q_{0}, \vec{q}, T\right) \\
& =-\frac{1}{\pi} \frac{\operatorname{Im} \Pi_{i}\left(q_{0}, \vec{q}, T\right)}{\left|q_{0}^{2}-\vec{q}^{2}-m_{i}^{2}-\Pi_{i}\left(q_{0}, \vec{q}, T\right)\right|^{2}} .
\end{aligned}
$$


For the case of pions, we incorporate the self-energy at finite temperature given in the Appendix of Ref. [35], which was obtained by incorporating the thermal effects to the $T=0$ pion self-energy model given, for instance, in Refs. [36,37]. We recall that the pion self-energy in nuclear matter at $T=0$ was obtained by adding to a small repulsive and constant $s$-wave part [38], the $p$-wave contribution coming from the coupling to $1 \mathrm{p}-1 \mathrm{~h}, 1 \Delta-1 \mathrm{~h}$, and $2 \mathrm{p}-2 \mathrm{~h}$ excitations together with short-range correlations. These correlations are mimicked by the LandauMigdal parameter $g^{\prime}$, taken from the particle-hole interaction described in Ref. [39], which includes $\pi$ and $\rho$ exchanges modulated by the effect of nuclear short-range correlations.

In the case of the $D(\bar{D})$ mesons, the self-energy is obtained self-consistently from the $s$-wave contribution to the in-medium $D(\bar{D}) \mathrm{N}$ amplitude, as will be shown explicitly at the end of this section.

With these medium modifications the propagator loop functions are obtained by four-momentum convolution of meson and baryon single-particle propagators:

$$
\begin{aligned}
G_{D(\bar{D}) N}\left(P_{0}, \vec{P}, T\right)= & \int \frac{d^{3} q}{(2 \pi)^{3}} \frac{M_{N}}{E_{N}(\vec{P}-\vec{q}, T)} \\
& \times\left[\int_{0}^{\infty} d \omega S_{D(\bar{D})}(\omega, \vec{q}, T)\right. \\
& \times \frac{1-n(\vec{P}-\vec{q}, T)}{P_{0}-\omega-E_{N}(\vec{P}-\vec{q}, T)+\mathrm{i} \varepsilon} \\
& +\int_{0}^{\infty} d \omega S_{\bar{D}(D)}(\omega, \vec{q}, T) \\
& \left.\times \frac{n(\vec{P}-\vec{q}, T)}{P_{0}+\omega-E_{N}(\vec{P}-\vec{q}, T)-\mathrm{i} \varepsilon}\right],
\end{aligned}
$$

for $D(\bar{D}) N$ states and

$$
\begin{aligned}
G_{\pi Y_{c}}\left(P_{0}, \vec{P}, T\right)= & \int \frac{d^{3} q}{(2 \pi)^{3}} \frac{M_{Y_{c}}}{E_{Y_{c}}(\vec{P}-\vec{q}, T)} \\
& \times \int_{0}^{\infty} d \omega S_{\pi}(\omega, \vec{q}, T) \\
& \times \frac{1+n_{\pi}(\vec{q}, T)}{P_{0}-\omega-E_{Y_{c}}(\vec{P}-\vec{q}, T)+\mathrm{i} \varepsilon},
\end{aligned}
$$

for $\pi \Lambda_{c}$ or $\pi \Sigma_{c}$ states, where $P=\left(P_{0}, \vec{P}\right)$ is the total two-particle four-momentum and $\vec{q}$ is the meson momentum in the nuclear matter rest frame. Note that, for the $D N$ loop function, the $S_{\bar{D}}(\omega, \vec{q})$ spectral function appearing in the subdominant second term on the right-hand side of Eq. (16) is assumed to be a free-space $\delta$ function. The $\pi Y_{(c)}$ loop function incorporates the $1+n_{\pi}(\vec{q}, T)$ term, with $n_{\pi}(\vec{q}, T)$ being the Bose distribution of pions at temperature $T$, to account for the contribution from thermal pions at finite temperature. Note that, by assuming perfect isospin symmetry, we set the pion chemical potential to zero in $n_{\pi}(\vec{q}, T)$.

For $\eta\left(\eta^{\prime}\right) Y_{c}, K \Xi_{c}\left(\Xi_{c}^{\prime}\right)$, and $D_{s} Y$ states, the corresponding meson lines (propagators) are not dressed by self-energy insertions. In the case of the $\eta, \eta^{\prime}$ mesons, this is a reasonable approximation, because the coefficients coupling the $\eta\left(\eta^{\prime}\right) Y_{c}$ channels with the $D N$ channel are small (see Tables I and II in Ref. [17]). Containing an $\bar{s}$ quark, the $K$ couples weakly to nucleons, and its spectral function may be approximated by the free space one, viz. by a $\delta$ function. We could include a moderate repulsive in-medium shift to the kaon mass, consistent with the repulsion predicted by a $T \rho$ approximation or more sophisticated models [40], but our results are insensitive to this shift due to the zero couplings of these channels to $D N$. As for the spectral function of the $D_{s}^{+}$meson appearing in the in-medium $D_{s} Y$ channels, it has been shown [24] that, in addition to the quasiparticle peak, it presents a lower energy mode associated with an exotic resonance predicted around $75 \mathrm{MeV}$ below the $D_{s}^{+} N$ threshold [23]. Therefore, with large coupling coefficients for transitions $D N \leftrightarrow D_{s} Y$, one may eventually have to solve an extended in-medium self-consistent coupled-channel problem combining the $C=$ $1, S=0(D N)$ and $C=1, S=1\left(D_{S} N\right)$ sectors. Work along this line is in progress.

Last, we state that the in-medium $D(\bar{D})$ self-energy is obtained by integrating $T_{D(\bar{D}) N}$ over the Fermi distribution for nucleon momentum at a given temperature as

$$
\begin{aligned}
\Pi_{D(\bar{D})}\left(q_{0}, \vec{q}, T\right)= & \int \frac{d^{3} p}{(2 \pi)^{3}} n(\vec{p}, T)\left[T_{D(\bar{D}) N}^{(I=0)}\left(P_{0}, \vec{P}, T\right)\right. \\
& \left.+3 T_{D(\bar{D}) N}^{(I=1)}\left(P_{0}, \vec{P}, T\right)\right],
\end{aligned}
$$

where $P_{0}=q_{0}+E_{N}(\vec{p}, T)$ and $\vec{P}=\vec{q}+\vec{p}$ are the total energy and momentum of the $D(\bar{D}) N$ pair in the nuclear matter rest frame and the values $\left(q_{0}, \vec{q}\right)$ stand for the energy and momentum of the $D(\bar{D})$ meson also in this frame. Recall that $\Pi_{D(\bar{D})}\left(q_{0}, \vec{q}, T\right)$ must be determined self-consistently because it is obtained from the in-medium amplitude $T_{D(\bar{D}) N}$ that contains the $D(\bar{D}) N$ loop function $G_{D(\bar{D}) N}$, and this last quantity itself is a function of $\Pi_{D(\bar{D})}\left(q_{0}, \vec{q}, T\right)$. From this we obtain the corresponding spectral function to complete the integral for the loop function $G_{D(\bar{D}) N}\left(P_{0}, \vec{P}, T\right)$ as in Eq. (16).

\section{RESULTS AND DISCUSSION}

\section{A. The $D$ meson spectral function in a hot nuclear medium}

We start this section by looking at the in-medium behavior of the $I=0 \Lambda_{c}$ and $I=1 \Sigma_{c}$ resonances, which in the full model of Ref. [17] appear at 2593 and $2770 \mathrm{MeV}$, respectively, in free space. This is summarized in Fig. 2 by the imaginary part of the in-medium $T(D N \rightarrow D N)$ matrix at saturation density $\rho_{0}=0.17 \mathrm{fm}^{-3}$ for $I=0$ (left column) and $I=1$ (right column) as a function of the center-of-mass energy $P_{0}$ for temperatures $T=0$ (first row) and $T=100 \mathrm{MeV}$ (second row), respectively. For each of the four figures, three different lines represent self-consistent calculations with increasing sophistications, viz. (i) the self-consistent dressing of $D$ mesons only (dotted lines), (ii) $D$-meson dressing with the inclusion of mean-field binding effect (MFB) on baryons in the loop functions (dash-dotted lines), and (iii) $D$-meson dressing with the inclusion of both baryon binding effects and pion dressing (PD) in the loop functions (solid lines). In the figures, the thick lines correspond to model A (viz. $\Sigma_{D N} \neq 0$ ), whereas the thin dashed lines refer to the result only for Case (iii) within model B $\left(\Sigma_{D N}=0\right)$. Recall that 


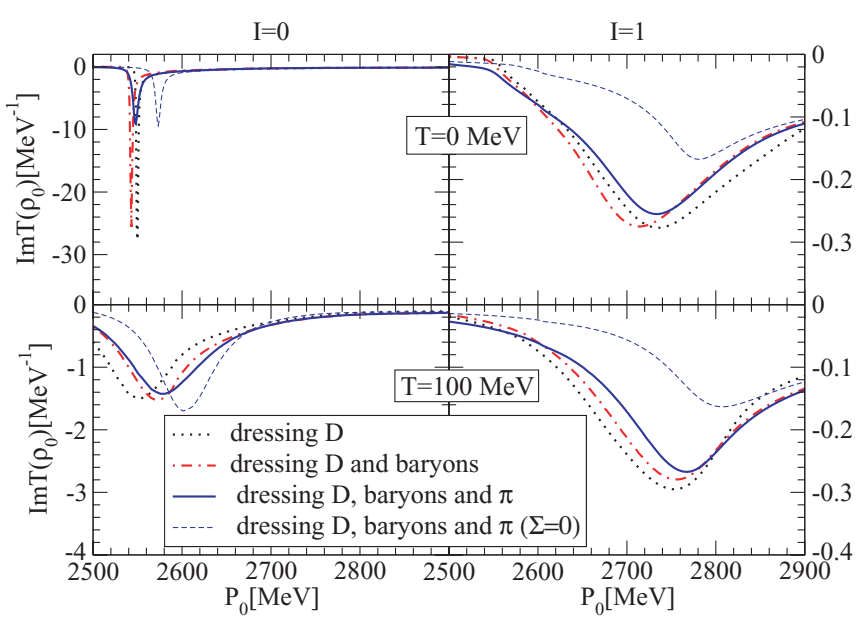

FIG. 2. (Color online) Imaginary part of the in-medium interaction for $I=0$ and $I=1$ at $\rho_{0}$ as a function of the centerof-mass energy $P_{0}$ for $T=0 \mathrm{MeV}$ and $T=100 \mathrm{MeV}$, and for three different approaches in the self-consistent calculation of the $D$ meson self-energy: (i) including only the self-consistent dressing of the $D$ meson, (ii) adding the binding of the different baryons in the intermediate states, and (iii) including the baryon binding effects and the pion self-energy.

our principal interest is in model A. Comparison of those two model results will be found later in this section.

Our discussion is first for the zero temperature $(T=0)$ case. We begin by comparing the two first cases. We recall that medium effects (excluding baryon binding potentials) lowered the position of the $\Lambda_{c}$ and $\Sigma_{c}$ resonances with respect to their free-space values [17]. When baryon binding effects are incorporated, these resonances get even more lowered, as can be seen by comparing the dotted and dash-dotted lines in Fig. 2. If we had a mere attractive shift of the nucleon mass of around $75 \mathrm{MeV}$ (the value of the optical potential at zero momentum) in a $D N$ single-channel (non-self-consistent) calculation, we would be expecting the same shift in the corresponding MFB amplitude. The fact that the attractive shifts induced by MFB effects are of only 6 and $22 \mathrm{MeV}$ for the in-medium $\Lambda_{c}$ resonance (hereafter denoted as $\tilde{\Lambda}_{c}$ ) and $\Sigma_{c}$ (denoted as $\tilde{\Sigma}_{c}$ ), respectively, indicates that coupled-channel effects, momentum dependence of the binding potentials, and self-consistency play crucial roles in the determination of the in-medium $D N$ amplitudes.

The widths of both resonances differ according to the phase space available. For the $I=0$ sector, the lowest threshold in free space is from the $\pi \Sigma_{c}$ channel, which lies slightly below the $\Lambda_{c}(2593)$ resonance position and constrains this resonance to be narrow. This is also the case in nuclear matter. This narrowness is somewhat relaxed by the processes $\tilde{\Lambda}_{c} N \rightarrow$ $\pi N \Lambda_{c}, \pi N \Sigma_{c}$, which open up in medium through the $D$-meson self-energy. The $I=1 \tilde{\Sigma}_{c}$ resonance, which shows a free-space width of $30 \mathrm{MeV}$ [17], develops a large width of the order of $200 \mathrm{MeV}$, due to the opening of new absorption processes of the type $\tilde{\Sigma}_{c} N \rightarrow \pi N \Lambda_{c}, \pi N \Sigma_{c}$, which is similar to the case of the $\tilde{\Lambda}_{c}$ but has a much larger decaying phase space.

As for the effect of pion dressing (PD) implemented in Case (iii), we expected it to be of minor importance in the present approach, in contrast to the findings in Refs. [27,35] for $\bar{K} N$ amplitude in nuclear matter and also in Refs. [21,22] for inmedium $D N$. This is due to the reduction factor $\kappa_{c} \approx 1 / 4$ in the $D N \leftrightarrow \pi Y_{c}$ transition [see Eq. (3)] due to the charm transfer as shown in Ref. [17]. Still, a small effect is seen in the positions and widths of the $\tilde{Y}_{c}\left(=\tilde{\Lambda}_{c}, \tilde{\Sigma}_{c}\right)$ through the absorption of these resonances by one and two nucleon processes $\left(\tilde{Y}_{c} N \rightarrow Y_{c} N\right.$ and $\left.\tilde{Y}_{c} N N \rightarrow Y_{c} N N\right)$, which open up through the $1 \mathrm{p}-1 \mathrm{~h}$ and $2 \mathrm{p}-2 \mathrm{~h}$ components of the pion self-energy.

With regard to the results for models $A$ and $B$, we observe that both models are qualitatively similar. However, the absence of the $\Sigma_{D N}$ term in model B produces in-medium resonances at higher energies, therefore the corresponding widths are larger due to the increased decaying phase space. As compared with their free-space resonance positions, the $\tilde{\Lambda}_{c}$ lies $45 \mathrm{MeV}$ lower and the $\tilde{\Sigma}_{c}$ is at $40 \mathrm{MeV}$ below the free value for model $\mathrm{A}$. In model $\mathrm{B}$, the $\tilde{\Lambda}_{c}$ lies $20 \mathrm{MeV}$ lower, whereas the $\tilde{\Sigma}_{c}$ moves up roughly by $10 \mathrm{MeV}$. These results are consistent with those obtained in Ref. [17] where meanfield baryon binding is absent. In the case of the $I=1$ amplitude, the attraction provided by a self-consistent calculation using model B is not enough to fully compensate the repulsion induced by Pauli blocking effects.

Now we come to the finite temperature case. The overall effects from $T \neq 0$ result is the reduction of the Pauli blocking factor because the Fermi surface is smeared out with temperature. Therefore, both resonances move up in energy to get closer to their position in free space, whereas they are smoothed out. The inclusion of MFB [viz. Case (ii)] for $T=100 \mathrm{MeV}$ induces a shift of both resonances to higher energies, opposite to what is found for $T=0$, hence it appears counterintuitive. The reason behind this is that the potential of each baryon from MFB becomes less attractive with increasing temperature and also with increasing momentum (see Fig. 1 for evolution in temperature of the baryonic potentials at zero momentum). At a high enough value of temperature, the single-particle potential may become repulsive already at a relatively low momentum. It will then become more difficult to excite intermediate states if they carry a repulsive potential and, consequently, the resonance will be generated at higher energies than in the absence of MFB effects, as is already the case at $T=100 \mathrm{MeV}$ and $\rho=\rho_{0}$. Again, the PD does not drastically alter the resonance positions. At $T=100 \mathrm{MeV}, \tilde{\Lambda}_{c}$ is at $2579 \mathrm{MeV}$ for model $\mathrm{A}$ and $\tilde{\Sigma}_{c}$ at $2767 \mathrm{MeV}$, whereas model B generates both resonances at higher energies: $\tilde{\Lambda}_{c}$ at $2602 \mathrm{MeV}$ and $\tilde{\Sigma}_{c}$ at $2807 \mathrm{MeV}$. The spreading of the resonant structures in $T_{D N}$ with increasing temperature has an important bearing in the temperature dependence of the $D$ meson spectral function as we shall discuss below.

In Fig. 3 we display the $D$ meson spectral function at zero momentum and normal saturation density $\rho_{0}$ for two distinct values of temperature ( $T=0$ and $T=100 \mathrm{MeV})$ and for Cases (i) to (iii) (thick lines) for model A. As in the previous figure, we only show the result from Case (iii) for model B with thin-dashed lines.

At $T=0$ the spectral function presents two peaks: the one at lower energy is built up from the $\tilde{\Lambda}_{c} N^{-1}$ excitation, whereas the second one at higher energy is mainly driven by the quasi $(D)$-particle peak but mixes considerably with the 


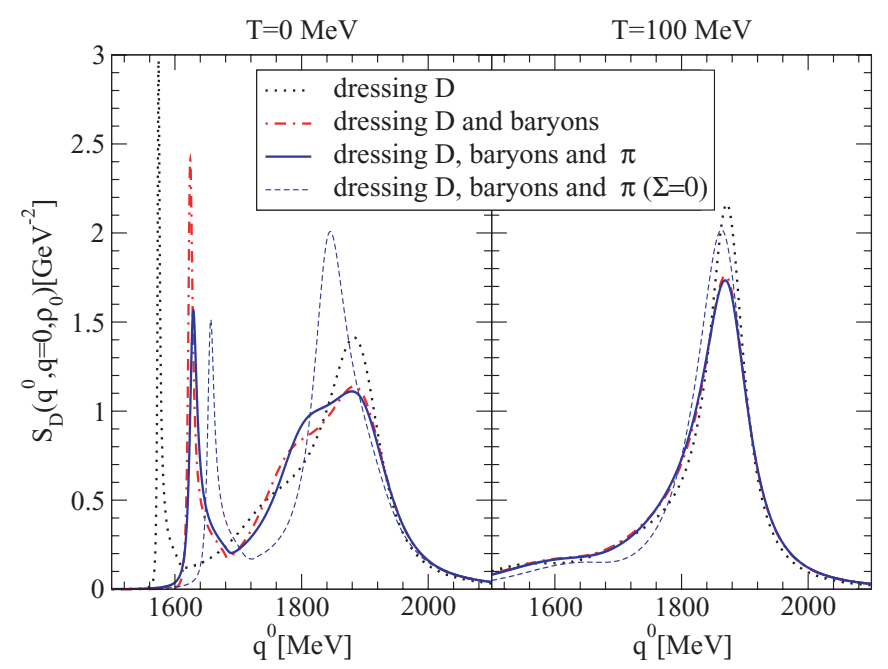

FIG. 3. (Color online) The zero-momentum $D$-meson spectral function at $\rho=\rho_{0}$ for $T=0 \mathrm{MeV}$ and $T=100 \mathrm{MeV}$ as a function of the $D$-meson energy for the previous approaches.

$\tilde{\Sigma}_{c} N^{-1}$ state. The quasiparticle energy $E_{q p}(\vec{q})$ may be found from the solution of $\operatorname{Re}\left[D_{D}\left(q_{0}, \vec{q}, T\right)\right]^{-1}=0$ for $q_{0}$, hence

$$
E_{q p}(\vec{q})^{2}=\vec{q}^{2}+m_{D}^{2}+\operatorname{Re} \Pi_{D}\left[E_{q p}(\vec{q}), \vec{q}\right] .
$$

We observe that, once MFB is included [Case (ii)], the lower peak in the spectral function due to the $\tilde{\Lambda}_{c} N^{-1}$ mode goes up by about $50 \mathrm{MeV}$ relative to the Case (i) result. This could be understood in the following manner: as seen in Fig. 2, the $\tilde{\Lambda}_{c}$ resonance moves to lower energies by about $6 \mathrm{MeV}$ upon going from Case (i) to Case (ii), but at the same time the nucleon energy goes down due to MFB, hence the peak in the $D$ meson spectral function goes up as the $\tilde{\Lambda}_{c} N^{-1}$ excitation effectively costs more energy. In other words, the meson requires to carry more energy to compensate for the attraction felt by the nucleon. The same characteristic feature is seen also for the $\tilde{\Sigma}_{c} N^{-1}$ configuration that mixes with the quasiparticle peak. Just in line with the in-medium $T_{D N}$ amplitude studied earlier (Fig. 2), the PD installed in Case (iii) does not alter much the position of $\tilde{\Lambda}_{c} N^{-1}$ excitation or the quasiparticle peak. From Eq. (19), the corresponding quasiparticle energy is found at $1855 \mathrm{MeV}$, i.e., lower than the free mass by $12 \mathrm{MeV}$. However, the actual peak appears slightly shifted upward due to the energy dependence of the imaginary part of the $D$-meson self-energy affected by the $\tilde{\Sigma}_{c} N^{-1}$ configuration. For model B [Case (iii) only], the absence of the $\Sigma_{D N}$ term moves the $\tilde{\Lambda}_{c} N^{-1}$ excitation closer to the quasiparticle peak, whereas the latter fully mixes with the $\tilde{\Sigma}_{c} N^{-1}$ excitation.

When the finite temperature effects are included (see the right-hand side of Fig. 3 for $T=100 \mathrm{MeV}$ ), the quasiparticle peak of the spectral function at zero momentum is found to move closer to the free-space mass value due to the smearing of the nuclear matter Fermi surface. The reason is that the selfenergy receives contributions from higher momentum $D N$ pairs that feel a weaker interaction. Furthermore, structures from the $\tilde{Y}_{c} N^{-1}$ modes seen at $T=0$ are smeared out with increasing temperature, an effect that was reported earlier in Ref. [22]. Eventually, at $T=100 \mathrm{MeV}$ we are left with a

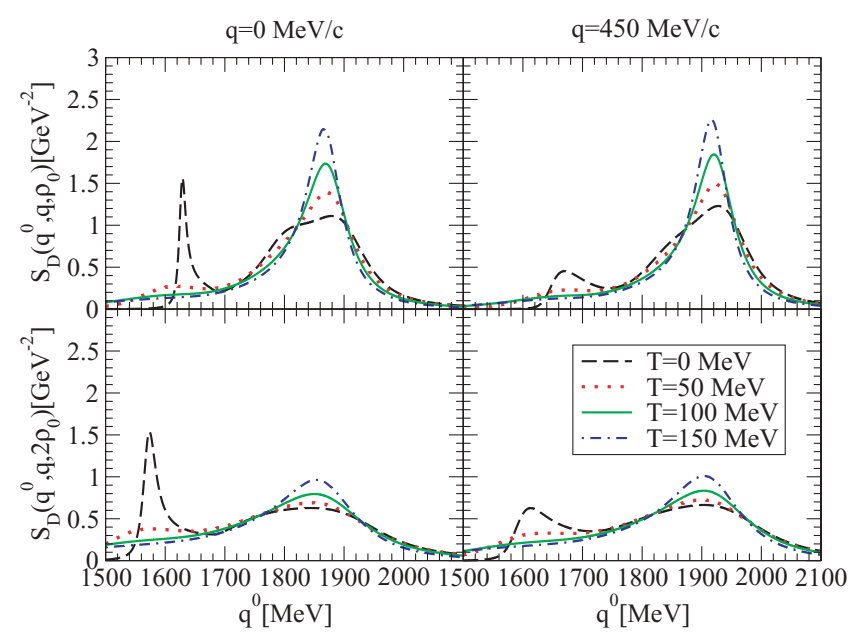

FIG. 4. (Color online) The $D$-meson spectral function for $q=0 \mathrm{MeV} / c$ and $q=450 \mathrm{MeV} / c$ at $\rho_{0}$ and $2 \rho_{0}$ as a function of the $D$-meson energy for different temperatures and for the self-consistent calculation, including the dressing of baryons and pions in model A $\left(\Sigma_{D N} \neq 0\right)$.

quasiparticle peak at $1869 \mathrm{MeV}$ (model A) and $1863 \mathrm{MeV}$ (model B), amazingly close to the free space mass, $M(D)=$ $1867 \mathrm{MeV}$, but with a large width due to collisional broadening. Again, due to the energy dependence of the self-energy, the positions of these peaks differ slightly from the value of the quasiparticle energies of 1864 and $1861 \mathrm{MeV}$, respectively. The slow falloff on the left-hand side of the quasiparticle peak corresponds mostly to the diluted $\tilde{\Lambda}_{c} N^{-1}$ configuration.

The evolution of the spectral function as a function of temperature is presented in Fig. 4 for two different densities, $\rho_{0}$ and $2 \rho_{0}$, and two momenta, $q=0 \mathrm{MeV} / c$ and $q=$ $450 \mathrm{MeV} / c$, in the full self-consistent calculation [Case (iii)] for model A. As already mentioned, we observe the dilution of the $\tilde{\Lambda}_{c} N^{-1}$ and $\tilde{\Sigma}_{c} N^{-1}$ structures with increasing temperature, whereas the quasiparticle peak gets closer to its free value and it becomes narrower. The widening of the quasiparticle peak for larger nuclear density may be understood as due to the enhancement of collision and absorption processes. As a result, the quasiparticle peak position is difficult to extract directly from the plot at high densities. As for the structure in the lower values of $q_{0}$ due to the $\tilde{\Lambda}_{c} N^{-1}$ configuration, it moves down with increasing nuclear matter density due to the lowering in the position of the $\tilde{\Lambda}_{c}$ resonance induced by the more attractive $\Sigma_{D N}$ term, as based on our experience in Ref. [17]. Then by picking up the case with $q=0, \rho=\rho_{0}$ (upper left-hand panel in Fig. 4) as an example, we want to analyze the behavior of the same structure as a function of $T$. This $\tilde{\Lambda}_{c} N^{-1}$ particle-hole configuration evolves from a sharp/narrow peak with very little strength below $q^{0}=$ $1600 \mathrm{MeV}$ for $T=0$ to a more diffused form at higher temperatures where it extends even below $q_{0}=1500 \mathrm{MeV}$. First, we see from the left-hand panel of Fig. 2 that the in-medium $I=0 \quad \tilde{\Lambda}_{c}$ resonance becomes broadened and shifted to higher energies with increasing temperature. We note, however, that a single nucleon-hole state may be created at a higher energy for higher $T$ because the nuclear matter Fermi surface gets more diffused. In addition, at higher $T$, the 
nucleon single-particle potential becomes repulsive already at relatively low momenta. Hence, the resulting $\tilde{\Lambda}_{c} N^{-1}$ configuration which dictates the $D$ spectral strength may spread eventually to lower energies as well with increasing temperature. So from what we see in Fig. 4, along with the wide collision broadening of the quasiparticle structure of $D$, this particle-hole structure as seen in the $D$ spectral function might well kinematically facilitate the decay process $J / \Psi N \rightarrow \tilde{\Lambda}_{c} \bar{D}$ in a dense nuclear matter at finite temperature, say, in high-energy heavy-ion collisions. This should of course depend on how the $\bar{D}$ meson may behave in the same nuclear matter environment, which we are studying below.

\section{B. $\bar{D}$ meson in nuclear matter}

With our models A and B introduced in Sec. II, we are able to study the properties of the $\bar{D}$ meson in a hot and dense nuclear matter. In fact, as found in Subsection 2.2, this case is far easier to deal with because the $\bar{D} N$ equation is a single-channel one for both $I=0$ and 1 isospin channels. Furthermore, the $\mathrm{T}-\mathrm{W}$ vector interaction in the $I=0$ channel has a strictly vanishing interaction strength, so the model $\mathrm{B}$ interaction produces no contribution in this isospin channel. In other words, the nonvanishing $I=0$ channel contribution in the $\bar{D} N$ channel comes entirely from the spin-isospin singlet $\Sigma_{D N}$ contribution in model A. The only available work to date on the $\bar{D}$ in nuclear matter is found in Ref. [24] for the case of zero $(T=0)$ temperature, with which we may compare our results.

We first present in Table I results for the effective $\bar{D} N$ interaction in free space. In particular, we show the $I=0$ and $I=1$ scattering lengths defined as

$$
a_{\bar{D} N}=-\frac{1}{4 \pi} \frac{M_{\bar{D} N}}{\sqrt{s}} T_{\bar{D} N \rightarrow \bar{D} N}
$$

at $\bar{D} N$ threshold, where $M_{\bar{D} N}$ is the total mass of the $\bar{D} N$ system. We use three different models that will be discussed in the following. The result from model A and B may not need any special explanation in view of what has been stated so far in the present subsection. However, we want to reiterate that the $I=0$ contribution in model A is entirely from the $\Sigma_{D N}$ contribution. On comparing these results with those from Ref. [24] $\left(a_{I=1}^{L K}=-0.26 \mathrm{fm}\right.$ and $\left.a_{I=0}^{L K}=-0.16 \mathrm{fm}\right)$ we see immediately that while the $I=1$ value is very close, there is a disagreement in the $I=0$ scattering length. In an attempt to clarify this discrepancy we have adopted a dimensional regularization method (DR) that was used in Ref. [24], but with some minor modification in the subtraction point as well as in the form of the interaction of Ref. [23], as discussed in Ref. [17]. The results, presented as the "DR" entries in Table I, are very close to the cut-off model B values, which

TABLE I. $\bar{D} N$ scattering lengths (fm)

\begin{tabular}{lccc}
\hline \hline & Model A & Model B & DR \\
\hline$I=0$ & 0.61 & 0 & 0 \\
(Born approx.) & $(0.26)$ & $(0)$ & $(0)$ \\
$I=1$ & -0.26 & -0.29 & -0.24 \\
(Born approx.) & $(-0.61)$ & $(-0.88)$ & $(-1.16)$ \\
\hline \hline
\end{tabular}

is what one should have anticipated given the fact that the modifications implemented in Ref. [17] alter the original form of the Hofmann-Lutz T-W interaction [24] and the unitarization of the amplitude only marginally, especially in the present $\bar{D} N$ channel. Note also that in our DR approach the value of the meson decay constant has been chosen to be $f=f_{\pi}$ as compared with $1.15 f_{\pi}$ in models $\mathrm{A}$ and $\mathrm{B}$. The $I=0$ scattering length turns out as, of course, zero, with the vanishing interaction strength. So the $a_{I=0}^{L K}$ value quoted in Ref. [24] remains to be somewhat puzzling to us.

A recent calculation of Haidenbauer and collaborators [41] employs a meson-exchange approach supplemented by a shortrange one-gluon exchange (OGE) contribution. It presents a similar $I=0$ scattering length $\left(a_{I=0}^{H}=-0.07 \mathrm{fm}\right)$ but the $I=1$ one is repulsive and almost twice our result $\left(a_{I=1}^{H}=\right.$ $-0.45 \mathrm{fm})$. Because the OGE mechanism has no counterpart within our model, the comparison of results may not be very meaningful at this point. Nevertheless, it is worth noting that about half of the repulsive scattering length $a_{I=1}^{H}$ comes from the hadronic meson-exchange contributions, which can be mapped to a certain extent to the $\mathrm{T}-\mathrm{W}$ interaction used in the present work.

To see whether the interaction is reasonably weak, hence the Born approximation be appropriate or not, we have also calculated the scattering lengths in that approximation as shown in the table. We find a big discrepancy between the exact and approximate values, thus concluding that one has to sum up the whole iterative series even for this apparently smooth $\bar{D} N$ interaction as noted also in Ref. [41]. Thus the Born approximation is not adequate in studying the in-medium $\bar{D}$ either. Note that, in the Born approximation, the $I=1$ scattering length is less repulsive in model A due to the attractive contribution of the spin-isospin singlet $\Sigma_{D N}$ term, whereas the scattering lengths calculated from the fully iterated amplitude are very similar in both models. Taking the isospin-averaged scattering length from the results in Table I, one establishes a repulsive nature for the $\bar{D} N$ interaction, even in model A, which contains the attractive effect of the $\Sigma_{D N}$ term.

The $\bar{D}$ optical potential in the nuclear medium may be defined as,

$$
U_{\bar{D}}(\vec{q})=\frac{\Pi_{\bar{D}}\left(E_{q p}(\vec{q}), \vec{q}\right)}{2 \sqrt{m_{\bar{D}}^{2}+\vec{q}^{2}}}
$$

and, at zero momentum, it can be identified as the in-medium shift of the $\bar{D}$ meson mass. Our results for the $\bar{D}$ mass shift are displayed in Fig. 5 in the case of model A (solid line) and B (dot-dashed line) including the MFB for nucleons.

The inclusion of an attractive $\Sigma_{D N}$ term in model A gives rise to a less repulsive mass shift at $\rho=\rho_{0}$, of $11 \mathrm{MeV}$, in contrast to the $20 \mathrm{MeV}$ repulsion found for model B. The absence of resonant states close to threshold in this $\bar{D} N$ scattering problem suggests extending the validity of the low-density theorem to normal nuclear matter densities or beyond. However, the low-density or $T \rho$ results, obtained by replacing the medium-dependent amplitude by the free-space one and displayed by the dashed and dotted lines for models $\mathrm{A}$ and $\mathrm{B}$, respectively, deviate quite substantially from the 


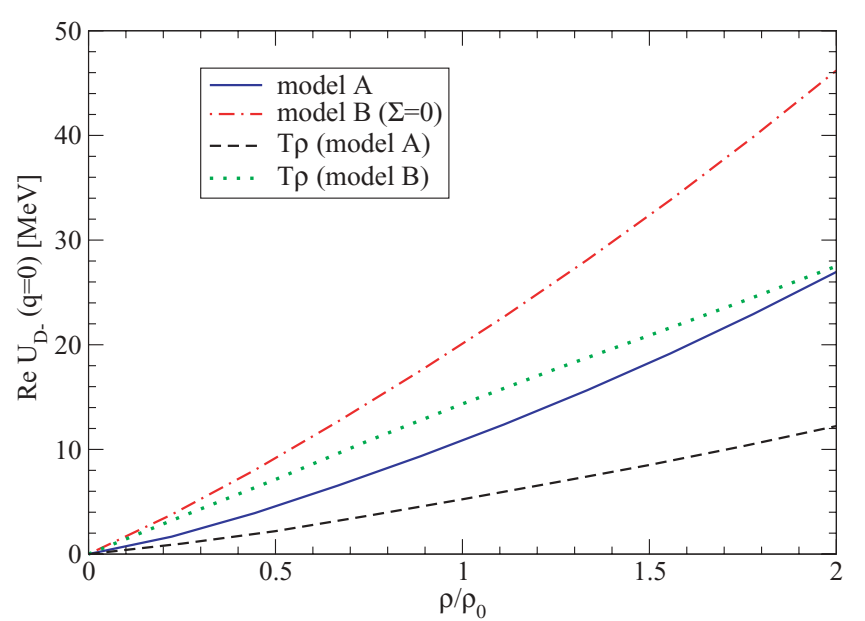

FIG. 5. (Color online) The $\bar{D}$ mass shift in model A and B $\left(\Sigma_{D N}=0\right)$, including the MFB for nucleons as well as the lowdensity approximation as a function of density.

corresponding fully self-consistent results at a relatively low value of nuclear matter density. The additional sources of density dependence present in a full calculation can also be visualized by the deviation of the solid and dot-dashed lines from a linear behavior. At normal nuclear matter density, the low-density mass shift for model B is $15 \mathrm{MeV}$, whereas the fully self-consistent result increases this value to $20 \mathrm{MeV}$. The same difference of about $5 \mathrm{MeV}$ between the mass shifts obtained in both approaches is found for model A, as can be seen in Fig. 5. Our mass shift of $20 \mathrm{MeV}$ at $\rho_{0}$ for model B is similar to the one in Ref. [24] within $10 \%$.

\section{In-medium $D$ and $\bar{D}$ optical potentials at finite temperature}

In this last subsection we compare in Fig. 6 the $D$ and $\bar{D}$ optical potentials at $q=0 \mathrm{MeV} / c$ as functions of temperature for two different densities $\left(\rho_{0}\right.$ and $\left.2 \rho_{0}\right)$ and for models $\mathrm{A}$ and B. The $D$-meson potential is calculated self-consistently with MFB on baryons and with PD, and the $\bar{D}$ meson is obtained
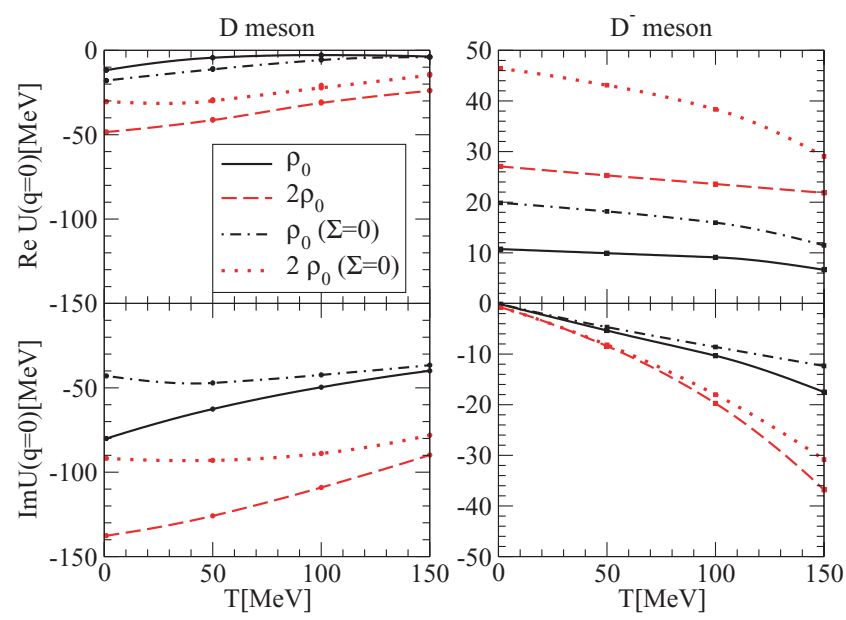

FIG. 6. (Color online) The $D$ and $\bar{D}$ potentials for the full self-consistent calculation at $q=0 \mathrm{MeV} / c$ for $\rho_{0}$ and $2 \rho_{0}$ in models $\mathrm{A}$ and $\mathrm{B}\left(\Sigma_{D N}=0\right)$ as a function of temperature. also in a self-consistent manner only with MFB, because pion dressing does not enter here. The mass shift for $D$ and $\bar{D}$ mesons is reduced with temperature because, as observed for the spectral functions in Fig. 4, the quasiparticle peak moves toward the free position. This effect was also observed previously in Ref. [22] and it is due to the reduction of the selfenergy as temperature increases because the meson-baryon interaction is averaged over larger momentum components where it is weaker. For model A (B) and at $T=0$, we obtain an attractive potential of $-12(-18) \mathrm{MeV}$ for $D$ meson, whereas the repulsion for $\bar{D}$ is 11 (20) MeV. A similar shift in the mass for $D$ mesons is obtained in Ref. [22]. The imaginary part and, hence, the width of the spectral function for the quasiparticle tends to increase slightly with temperature for $\bar{D}$ mesons due to the increase in the collisional width, whereas for $D$ mesons it is somewhat reduced. Note, however, the different energy scales used in the $D$ and $\bar{D}$ plots. In fact, the situation is more involved for $D$ mesons. On the one hand, the collisional width due to $D N \rightarrow D N$ processes also increases with temperature, but at low $T$ the $D$ meson width is largely dominated by the mixing of the quasiparticle peak to the $\tilde{\Sigma}_{c} N^{-1}$ components of the $D$ meson self-energy. This is also the reason why the quasiparticle peak is located at a lower energy for model B, contrary to what one expects, as explained in the subsequent paragraph below. As $T$ increases, the $\tilde{\Sigma}_{c}$ resonance gets diluted and, correspondingly, the width decreases. It is expected, however, that the width will eventually increase with $T$ when it becomes mostly of collisional origin at high-enough temperatures.

With regard to the effect of the $\Sigma_{D N}$ term, we find that, for $\bar{D}$ mesons, its inclusion substantially reduces the repulsion, independently of the temperature and density, because the dominant repulsive $I=1$ scattering length is partly compensated by the attractive $I=0$ one. However, this simple picture cannot be applied to the $D$ meson due to the presence of the $I=1 \tilde{\Sigma}_{c}$ close to the $D N$ threshold. We see that, in this case, there is a crossover when we go from $\rho_{0}$ to $2 \rho_{0}$. The inclusion of the $\Sigma_{D N}$ term alters the position of the $\tilde{\Sigma}_{c}$ close to the $D N$ threshold (as seen in Fig. 2). Therefore, while it has the expected attractive effect to the real part of the potential at $2 \rho_{0}$, it effectively induces a repulsive effect for $\rho_{0}$. The imaginary part increases with the inclusion of $\Sigma_{D N}$ term for both mesons.

\section{SUMMARY AND CONCLUSIONS}

We have performed a hadronic self-consistent coupledchannel calculation of the $D$ and $\bar{D}$ self-energies in symmetric nuclear matter at finite temperature taking an effective mesonbaryon Lagrangian that combines the charmed meson degree of freedom in a consistent manner with the chiral unitary models. This interaction consists of a broken $s$-wave SU(4) T-W contribution supplemented by a scalar-isoscalar $\Sigma_{D N}$ term interaction. The corresponding in-medium solution at finite temperature obtains the dressing of $D(\bar{D})$ by Pauli blocking effects, dressing of $\pi$ (PD), and the nuclear mean field binding effect (MFB) not only on the nucleons but also on the charmed and strange hyperons by a finite-temperature $\sigma-\omega$ mean-field calculation.

In nuclear matter at $T=0$, the dynamically generated $I=$ $0 \tilde{\Lambda}_{c}$ and $I=1 \tilde{\Sigma}_{c}$ resonances in the $C=1$ charm sector lie 
around $40 \mathrm{MeV}$ below their free space values. Also at $T=0$, the baryon binding results in an attractive mass shift for those resonances as compared to the case with no such effect. But, as we incorporate finite temperature, those resonances tend to move back to their free position acquiring a remarkable width due to the smearing of the Fermi surface.

The $\tilde{\Lambda}_{c}$ and $\tilde{\Sigma}_{c}$ resonances induce resonant-hole excitation modes that are clearly seen in the low-temperature $D$-meson spectral function. The width of the distribution in fact reflects the overlap of the quasiparticle peak with the $\tilde{\Sigma}_{c} N^{-1}$ components of the $D$-meson self-energy. As temperature increases, these modes tend to smear out and the $D$-meson spectral function becomes a single pronounced quasiparticle peak close to the free $D$-meson mass with fairly extended tails, particularly to the lower-energy side of the distribution. At high temperature the width of the quasiparticle peak gets reduced slightly, so most of the distribution of the spectral function concentrates around the quasiparticle energy, although maintaining the overall strength in its lower energy part. As density increases, the quasiparticle peak broadens and the low-energy strength, associated to the $\tilde{\Lambda}_{c} N^{-1}$ components and related to $Y_{c} \pi N^{-1}, Y_{c} N N^{-2}, \ldots$ absorption modes, obviously increases.

In the $\bar{D} N$ sector, we have first obtained the free space $I=0$ and $I=1$ scattering lengths. While our repulsive $I=1$ value of $a_{I=1} \sim-0.3 \mathrm{fm}$ is in good agreement with Lutz and Korpa results [24], the finite value for the $I=0$ scattering length found in this latter reference is in contrast to the zero value found here for model $\mathrm{B}$ due to the vanishing $I=0$ coupling coefficient of the corresponding pure T-W $\bar{D} N$ interaction. Our results are, however, consistent with a recent calculation based on a meson-exchange model supplemented by a shortrange one-gluon exchange contribution [41]. For model A, we obtain a nonzero value of the $I=0$ scattering length, dictated entirely by the magnitude of the $\Sigma_{D N}$ term, which takes a rather conservative value in our present work. We have also observed that, in spite of the weakness of the $\bar{D} N$ interaction and the absence of resonances close to threshold, the Born approximation is not sufficient to describe the free-space $\bar{D} N$ interaction at low energies.

As for medium effects, they induce a repulsive shift in the $\bar{D}$ meson mass of $11 \mathrm{MeV}(20 \mathrm{MeV})$ for model A (B) in nuclear matter at saturation density. Although the medium modifications of the $\bar{D} N$ interaction are more moderate than in the case of $D N$, we observe that the low-density approximation breaks down at relatively low densities. At nuclear matter saturation density, the $\bar{D}$-meson mass shifts obtained from a fully self-consistent calculation are $5 \mathrm{MeV}$ larger than those of the low-density approximation. The temperature dependence of the repulsive real part of the $\bar{D}$ optical potential is very weak, whereas the imaginary part increases steadily due to the increase of collisional width. The picture is somewhat different for the $D$ meson. At low temperature, the corresponding quasiparticle peak is already quite broad due to the overlap with the $\tilde{\Sigma}_{c} N^{-1}$ mode. As temperature increases the later mode tends to dissolve and, with the overlap being reduced, one observes an overall decrease in the width of the distribution in spite of the increase of collisional broadening.
Taking into account our results, we might look at the question of possible $\bar{D}$ bound states once discussed in Ref. [13]. While $D^{-}$-mesic nuclei systems will always be bound by the Coulomb interaction, it would be interesting to see whether strongly bound nuclear states or even bound $\bar{D}^{0}$ nuclear systems might exist. From the results of Fig. 5 we see that, even for model $\mathrm{A}$, the $\bar{D}$-nucleus optical potential at zero momentum is repulsive, hence ruling out this possibility. However, as mentioned earlier, the $\Sigma_{D N}$ term, which contributes attractively to both isospin channels of the $\bar{D} N$ interaction, has been given a conservative value in our present approach. Although the magnitude of the $\Sigma_{D N}$ term may be made larger, we recall that it is not a free parameter but is constrained by the coupled $D N$ channel, in particular by the properties of the $\Lambda_{c}(2593)$ resonance. Therefore, it will be first necessary to see whether a larger $\Sigma_{D N}$ term constrained by the properties of the $D N$ interaction may still produce an attractive isospin averaged interaction, which could then even allow for the existence of $\bar{D}^{0}$-nucleus bound states. In any event, it is clear that $D^{-}$-mesic nuclei provide a valuable source of information for determining the sign and size of the $\bar{D}$ mass shift at subnuclear densities. An experimental observation of bound $D$ nuclear states is ruled out by the large width and moderate attraction found for the $D$-meson optical potential.

The other point of interest from the present study is related to the possible hadronic mechanism for the suppression of the $J / \Psi$ production in relativistic heavy-ion collisions. As stated in the introduction, a good part of the earlier interest was to see if the masses of $D$ and $\bar{D}$ get reduced so that the $J / \Psi \rightarrow D \bar{D}$ may proceed exothermically $[13,14,16,24]$ in nuclear matter, hence contributing to the $J / \Psi$ suppression. The difference in the two thresholds in vacuo for this process is $\Delta E \equiv E_{\mathrm{th}}(D+\bar{D})-E_{\mathrm{th}}(J / \Psi) \approx 650 \mathrm{MeV}$, which should be overcome in one way or another for this to go spontaneously or at the cost of small energies. Because charmonia, including the $J / \Psi$, are $c \bar{c}$ bound states that contain no light quarks, one normally assumes that the medium modification they might undergo should be minimum, thus their in-medium masses are not expected to be very different from those in vacuo. In the present work, we have observed that in-medium $\bar{D}$ mass increases typically by 10-20 MeV. On the other hand, as mentioned in the last section, from Fig. 4, the tail of the quasiparticle peak of the $D$ spectral function extends with a non-negligible strength to lower "mass" values due to the thermally spread $\tilde{Y}_{c} N^{-1}$ particle-hole configurations. So one might expect that some spontaneous leakage for $J / \Psi N \rightarrow$ $\tilde{Y}_{c} \bar{D} \rightarrow Y_{c} \pi \bar{D}, J / \Psi N N \rightarrow Y_{c} N \bar{D}, \ldots$ might effectively be possible. However, it looks very unlikely that the lower part of this spectral function tail extends from the quasiparticle peak as far down by $600 \mathrm{MeV}$ while keeping relevant strengths. So the disappearance of the $J / \Psi$ through such processes, even if helped by the thermally excited nucleons, will not proceed. These processes might still go endothermically through collisions induced by a surrounding large collection of comoving hadrons for which the effective reaction threshold may get lowered. But its efficiency is very questionable. So the direct disappearance of the existing $J / \Psi$ looks improbable.

Then, what about the possibility of reducing the supply of this charmonium from its excited state partners 
such as $\chi_{c \ell}(1 P),(\ell=0,1,2)$ because it is known that an appreciable fraction of $J / \Psi$ production comes from the radiative decay of these charmonia [42]? To look for such a possibility, we may consider here the following reactions: $\chi_{c \ell}(1 P) N \rightarrow \Lambda_{c}(2285) \bar{D}, \chi_{c \ell}(1 P) N \rightarrow$ $\Lambda_{c}(2285) \pi \bar{D}, \chi_{c \ell}(1 P) N \rightarrow \Sigma_{c}(2445) \bar{D}$, and $\chi_{c \ell}(1 P) N \rightarrow$ $\tilde{\Lambda}_{c}(2593) \bar{D}$. Note that the masses of these $\chi_{c \ell}$ 's are 3415,3511 , and $3556 \mathrm{MeV}$ in ascending order for $\ell$. Before proceeding, we note that in free space the first three reactions are already endothermic for all three $\chi_{c \ell}$ 's, whereas the last one is closed for the two lowest ones. On taking into account the MFB effects studied in the present work, it should be safe to speculate that the same first three reactions do take place in a hot nuclear matter as well. As for the last one, we might also claim that it could proceed in medium because the $\tilde{\Lambda}_{c}$ will develop a sufficient width as seen, for example, in the in-medium $D N$ amplitudes of Fig. 2 or reflected in the extended low-energy tail of the $D$-meson spectral functions in Fig. 4. Therefore part of the feeding of the $J / \Psi$ from its excited state partners will certainly be reduced. A similar mechanism might well reduce the feeding of $J / \Psi$ from the decay of the $\Psi^{\prime}$. The arguments presented here are only kinematical in nature, so even a simple dynamical model for the relevant in-medium reactions may need to be employed to support them further.

\section{ACKNOWLEDGMENTS}

T. M. is grateful to the support offered through the host (A. R.) for his stay in Barcelona, and for part of his travel. This work is partly supported by the EU contract FLAVIAnet MRTN-CT-2006-035482, by the contract FIS2005-03142 from MEC (Spain) and FEDER and by the Generalitat de Catalunya contract 2005SGR-00343. This research is part of the EU Integrated Infrastructure Initiative Hadron Physics Project under contract number RII3-CT-2004-506078. L. T. wishes to acknowledge support from the BMBF project "Hadronisierung des QGP und Dynamik von Hadronen mit Charm Quarks" (ANBest-P and BNBest-BMBF 98/NKBF98).
[1] T. Matsui and H. Satz, Phys. Lett. B178, 416 (1986).

[2] M. Gonin et al. (NA50 Collaboration), Nucl. Phys. A610, 404c (1996).

[3] L. Lammello et al. (NA50 Collaboration), Nucl. Phys. A638, 261c (1998); M. C. Abreu et al., Phys. Lett. B450, 456 (1999).

[4] M. C. Abreu et al., Eur. Phys. J. C 14, 443 (2000).

[5] R. Arnaldi et al. (NA60 Collaboration), J. Phys. G: Nucl. Part. Phys. 32, S51 (2006).

[6] A. Adare et al. (PHENIX Collaboration), Phys. Rev. Lett. 98, 232301 (2007); T. Gunji (PHENIX Collaboration), J. Phys. G: Nucl. Part. Phys. 34, S749 (2007).

[7] Y. Aoki, Z. Fodor, S. D. Katz, and K. K. Szabo, Phys. Lett. B643, 46 (2006).

[8] Z. Fodor and S. D. Katz, J. High Energy Phys. 04 (2004) 050.

[9] C. Ratti, M. A. Thaler, and W. Weise, Phys. Rev. D 73, 014019 (2006).

[10] K. Tsushima, A. Sibirtsev, K. Saito, A. W. Thomas, and D. H. Landau, Nucl. Phys. A680, 280 (2001).

[11] W. Cassing, E. L. Bratkovskaya, and A. Sibirtsev, Nucl. Phys. A691, 753 (2001).

[12] R. Shahoyan (NA60 Collaboration), J. Phys. G: Nucl. Part. Phys. 34, S1029 (2007).

[13] K. Tsushima, D. H. Lu, A. W. Thomas, K. Saito, and R. H. Landau, Phys. Rev. C 59, 2824 (1999).

[14] A. Sibirtsev, K. Tsushima, and A. W. Thomas, Eur. Phys. J. A 6, 351 (1999).

[15] A. Hayashigaki, Phys. Lett. B487, 96 (2000).

[16] A. Mishra, E. L. Bratkovskaya, J. Schaffner-Bielich, S. Schramm, and H. Stöcker, Phys. Rev. C 70, 044904 (2004).

[17] T. Mizutani and A. Ramos, Phys. Rev. C 74, 065201 (2006).

[18] M. Gazdzicki and M. I. Gorenstein, Phys. Rev. Lett. 83, 4009 (1999).

[19] M. Gazdzicki, Phys. Rev. C 60, 054903 (1999).

[20] K. Gallmeister, B. Kämpfer, and O. P. Pavelenko, Phys. Lett. B473, 20 (2000).

[21] L. Tolos, J. Schaffner-Bielich, and A. Mishra, Phys. Rev. C 70, 025203 (2004).
[22] L. Tolos, J. Schaffner-Bielich, and H. Stöcker, Phys. Lett. B635, 85 (2006).

[23] J. Hofmann and M. F. M. Lutz, Nucl. Phys. A763, 90 (2005).

[24] M. F. M. Lutz and C. L. Korpa, Phys. Lett. B633, 43 (2006).

[25] J. Oller and U. G. Meissner, Phys. Lett. B500, 263 (2001).

[26] E. Oset and A. Ramos, Nucl. Phys. A635, 99 (1998).

[27] A. Ramos and E. Oset, Nucl. Phys. A671, 481 (2000).

[28] L. Tolos, A. Ramos, and E. Oset, Phys. Rev. C 74, 015203 (2006).

[29] T. Maruyama, T. Muto, T. Tatsumi, K. Tsushima, and A. W. Thomas, Nucl. Phys. A760, 319 (2005).

[30] M. Artuso et al. (CLEO collaboration), Phys. Rev. Lett. 95, 251801 (2005).

[31] W. M. Yao et al. (Particle Data Group), J. Phys. G: Nucl. Part. Phys. 33, 1 (2006).

[32] J. I. Kapusta and C. Gale, Finite Temperature Field Theory Principles and Applications, 2nd ed. (New York, Cambridge University Press, 2006).

[33] R. Machleidt, Adv. Nucl. Phys. 19, 189 (1989).

[34] K. Tsushima and F. C. Khanna, Phys. Lett. B552, 138 (2003); K. Tsushima and F. C. Khanna, J. Phys. G: Nucl. Part. Phys. 30, 1765 (2004).

[35] L. Tolos, A. Ramos, and A. Polls, Phys. Rev. C 65, 054907 (2002).

[36] E. Oset, P. Fernandez de Cordoba, L. L. Salcedo, and R. Brockmann, Phys. Rep. 188, 79 (1990).

[37] A. Ramos, E. Oset, and L. L. Salcedo, Phys. Rev. C 50, 2314 (1994).

[38] R. Seki and K. Masutani, Phys. Rev. C 27, 2799 (1983); O. Meirav, E. Friedman, R. R. Johnson, R. Olszewski, and P. Weber, ibid. 40, 843 (1989).

[39] E. Oset, H. Toki, and W. Weise, Phys. Rep. 83, 28 (1982).

[40] L. Tolos, D. Cabrera, A. Ramos, and A. Polls, Phys. Lett. B632, 219 (2006); and references herein.

[41] J. Haidenbauer, G. Krein, U. G. Meissner, and A. Sibirtsev, Eur. Phys. J. A 33, 107 (2007).

[42] L. Antoniazzi et al. (E705 Collaboration), Phys. Rev. Lett. 70, 383 (1993). 